\title{
Contextos de Aprendizaje en el Nivel Medio y en la Universidad. Percepciones y Expectativas de Estudiantes que Ingresan en Carreras de Ingeniería
}

\author{
Paola V. Paoloni(1), Rocío B. Martín ${ }^{(2)}$ y Analía C. Chiecher ${ }^{(3)}$ \\ (1) Facultad de Ingeniería y Facultad de Ciencias Humanas de la Universidad Nacional de Río Cuarto, Ruta \\ Nacional 36, km 601, Ciudad de Río Cuarto, provincia de Córdoba, República Argentina \\ (e-mail: paopaoloni17@hotmail.com) \\ (2) Facultad de Ciencias Humanas de la Universidad Nacional de Río Cuarto, Ruta Nacional 36, km 601, \\ Ciudad de Río Cuarto, provincia de Córdoba, República Argentina (e-mail: rociobelenmartin@gmail.com) \\ (3) Facultad de Ingeniería de la Universidad Nacional de Río Cuarto, Ruta Nacional 36, km 601, Ciudad de \\ Río Cuarto, provincia de Córdoba, República Argentina (e-mail: achiecher@hotmail.com)
}

Recibido Ene. 14, 2015; Aceptado Mar. 19, 2015; Versión final May. 23, 2015, Publicado Dic. 2015

\begin{abstract}
Resumen
En este trabajo se exponen y analizan las principales características y resultados de un estudio orientado a conocer las percepciones y expectativas sobre contextos de aprendizaje de un grupo de estudiantes que ingresan en carreras de ingeniería. A inicio del ciclo lectivo 2014, se trabajó con 137 aspirantes a cursar alguna de las carreras que dicta la Facultad de Ingeniería de la Universidad Nacional de Río Cuarto (Argentina). Los datos fueron recabados por medio del Inventario sobre el Ambiente de Estudio Percibido (Inventory of Perceived Study Environment). Los resultados muestran diferencias entre las percepciones de los estudiantes sobre los contextos de aprendizaje de sus colegios de educación secundaria y las expectativas sobre los contextos de aprendizaje que les gustaría encontrar en la universidad. Los hallazgos de este trabajo son útiles para el diseño e implementación de contextos instructivos que favorezcan la integración y la permanencia de los alumnos que ingresan a la universidad.
\end{abstract}

Palabras clave: aspirantes a ingeniería, contextos de aprendizaje, percepciones de aprendizaje

\section{Learning Contexts from at High School and University Level. Perceptions and Expectations of Freshmen in Engineering Careers}

\begin{abstract}
The main features and results of a project developed to understand the perceptions and expectations about learning contexts of a group of freshmen in engineering careers are presented and analyzed. At the beginning of the school year 2014, a sample of 137 freshmen student of the careers offered by the Faculty of Engineering of the Universidad Nacional de Río Cuarto (Argentina) was selected for study. Data were collected using the Inventory of Perceived Study Environment. The results show differences between the perceptions of students on the learning contexts of their high schools and the expectations for the learning contexts that they would like to find at the university. The findings of this study are useful for the design and implementation of instructional contexts that encourage integration and retention of new students entering the university.
\end{abstract}

Keywords: engineering freshmen, learning contexts, learning perceptions, university 


\section{INTRODUCCIÓN}

Según Vélez (2005), sólo el 16,1\% de la población argentina comprendida entre los 18 y los 24 años de edad accede a los estudios superiores. La autora advierte que si comparamos este porcentaje con cifras internacionales se pone en evidencia una tasa de incorporación muy baja. La preocupación por atender al ingreso y a la permanencia de los estudiantes parece más acuciante si la consideramos en relación con las carreras de ingeniería. Si bien en este trabajo se emplea el uso del masculino como genérico para referir a alumnos y docentes, ello no debe interpretarse como 'leguaje sexista' que intenta discriminar a las mujeres. Nuestra decisión se fundamenta en la intención de contribuir a una lectura más amena, comprensible y focalizada entorno a los objetivos que estructuran el estudio. El abandono de los estudios, como antítesis de la permanencia deseada, es un hecho que golpea duramente a las ingenierías. La escasez de ingenieros comienza a ser un problema en todo el mundo. El desarrollo de la ciencia, el rápido crecimiento y diversificación tecnológica y la generalización de la informática, exigen una densidad de conocimiento técnico que no parece cubrirse con profesionales de estas especialidades por el escaso número de graduados con que se cuenta (Panaia, 2007). En Argentina, especialistas e investigadores en educación, autoridades de instituciones de enseñanza universitaria y políticos en general, han mostrado una fuerte preocupación por el curso de las ingenierías (Panaia, 2011a).

Las carreras de ingeniería en Argentina se caracterizan en general por un decreciente número de inscriptos, un reducido número de graduados, la lentificación en el recorrido de los trayectos curriculares -que profundiza la brecha entre la duración real de las carreras y la duración teórica que figura en los planes de estudio- y un marcado abandono de los estudios que en general ocurre durante los primeros meses de cursada (García et al., 2011). El porcentaje de estudiantes que abandonan sus estudios en carreras de Ingeniería en todo el país, oscila entre el 30\% y el 50\% (Panaia, 2011b). Por otra parte, el promedio de ingenieros especialistas que se gradúa por año es muy bajo. Así, en Argentina se gradúan por año unos 56 ingenieros por cada millón de habitantes, mientras que en países como Corea se gradúan unos 1187 ingenieros por año y por millón de habitantes. Si comparamos nuestra situación con la de países latinoamericanos, el panorama sigue resultando poco alentador. En este sentido, naciones como Colombia o México producen, respectivamente, 261 y 226 ingenieros por año y por millón de habitantes, no logrando incluso cubrir sus demandas internas (Panaia, 2007).

Apoyar la incorporación y la permanencia de los estudiantes en la educación superior, y específicamente en carreras de ingeniería, es una urgencia a la que debemos atender, no sólo desde políticas estatales o acciones ministeriales, sino y principalmente, desde el lugar en el que como profesionales nos desempeñamos día a día. El trabajo que presentamos a continuación fue pensado e implementado desde nuestro lugar como investigadoras educacionales en la Facultad de Ingeniería de la Universidad Nacional de Río Cuarto. Se funda en la hipótesis según la cual el análisis de las condiciones de partida de los estudiantes que ingresan -heterogéneas sin duda-, puede echar luces en el desafío de diseñar contextos instructivos promisorios para el aprendizaje. Suponemos que mientras más conozcamos acerca de las vivencias de los alumnos durante sus trayectorias como estudiantes del nivel medio y más sepamos acerca de sus expectativas acerca de los contextos de aprendizaje que les gustaría encontrar en el nivel universitario, más y mejores herramientas tendremos para el diseño de situaciones de aprendizajes potentes en sus posibilidades para promover en ellos mayor interés, motivación y aprendizajes significativos.

Junto a la difusión de las aportaciones de las teorías socio-constructivistas en el campo de la Psicología Educacional, los estudios sobre los contextos de aprendizajes recibieron una atención creciente en los últimos años (Bransford et al., 2003; Cole, 1999; De Corte, 2000). En este marco, Cole (1999) destacó la frecuencia con que los términos hebras, hilos, tejidos, red, se usan dentro de los enfoques culturalistas para describir acciones en el estudio de los procesos de pensamiento. Cuando el contexto se entiende como una red y las acciones se estudian como acontecimientos particulares, los vínculos entre la persona, las demandas de la tarea, los propósitos de la acción y las herramientas materiales o simbólicas disponibles, pasan a ser objeto de mayor interés. En este marco, los aprendizajes individuales se consideran inseparables de la construcción colectiva del conocimiento; el aprendizaje se entiende como un proceso social y situado, basado en la interacción y cooperación entre las personas (Rinaudo, 2014).

Las percepciones de los estudiantes sobre el ambiente de aprendizaje pueden pensarse como el resultado de la interacción entre comportamientos y rasgos individuales y las influencias del contexto de aprendizaje (Könings et al., 2005a). Los estudiantes perciben e interpretan los contextos a partir de las concepciones que van formando sobre el aprendizaje y las tareas que se van desarrollando durante el mismo. Todos los actores del proceso educativo -profesores y alumnos- tienen su propia perspectiva sobre el proceso de aprendizaje y, al parecer, una mayor congruencia entre tales interpretaciones favorecería la concreción de aprendizajes más efectivos. La percepción de los estudiantes sobre el ambiente de aprendizaje influiría 
entonces en la realización de las actividades y en la calidad de los desempeños y resultados obtenidos (Könings et al., 2005a).

Las ideas formuladas hasta aquí destacan la relevancia de atender a las percepciones de los estudiantes en el diseño de un contexto de aprendizaje. En relación a ello, un estudio de Könings et al. (2005b) ha mostrado diferencias significativas entre las percepciones de los estudiantes acerca del aprendizaje y las percepciones construidas por los profesores. Así, en esta investigación en particular, se ha demostrado que los estudiantes tenían una visión más negativa que sus profesores en relación con las metas y el aprendizaje; influyendo en consecuencia en su predisposición para comprometerse en las diferentes asignaturas.

Para este trabajo, nuestra consideración de los aspectos interpersonales que dan vida a cada comunidad de aprendizaje, refirió principalmente a la participación de los alumnos aspirantes en la construcción de sus identidades como estudiantes y en metas personales y académicas compartidas. Si el contexto es considerado metafóricamente como 'algo que entrelaza', 'algo que envuelve' (Cole, 1999), entonces el estudio de las percepciones de los ingresantes y de sus expectativas acerca de los contextos de aprendizaje, brindaría una contribución importante para pensar en contextos instructivos que tengan en cuenta estas identidades y proyecciones en el tiempo. Así, las experiencias que unen a los aspirantes a carreras de ingeniería como grupo y que los distinguen como personas, y el conocimiento de las expectativas académicas que proyectan hoy de cara hacia el mañana, constituyen elementos a tener en cuenta para pensar en diseños instructivos capaces de promover la conformación de comunidades de aprendizaje durante la etapa de ingreso en la universidad.

El ingreso en la universidad constituye una situación particularmente crítica en la que confluyen múltiples variables personales y contextuales, en constante estructuración y re-estructuración. Para muchos estudiantes, ingresar en la universidad exige aprendizajes que van más allá de lo académico y lo institucional. Se trata de aprender los códigos, rituales, rutinas, de una nueva cultura, de un nuevo contexto, que en principio no resulta familiar (Paoloni y Moreno, 2013; Vélez, 2005).

Ingresar en la universidad implica cambios y todo cambio requiere de reorganizaciones, de adaptaciones en lo personal, en lo familiar y en lo social. Estos procesos dinámicos vinculados con el ingreso en la universidad, se dan en plena adolescencia, una etapa de la vida caracterizada por la restructuración de la propia identidad, lo que implica, entre otros aspectos, redefinir metas, autopercepciones e incluso las valoraciones de nuestras potencialidades. Así, interrogantes como ¿qué me gustaría lograr a través de una carrera?, ¿con qué herramientas cuento?, ¿qué aprendizajes necesito construir?, ¿qué recursos del contexto pueden ayudarme?, etc., nos motivan a reflexionar sobre nosotros mismos y sobre el contexto (Luján et al., 2004).

Si el primer año universitario constituye un momento crucial en la vida de cualquier estudiante y si, por esto mismo, los espacios de reflexión sobre sí mismos y sobre el contexto se tornan necesarios durante esta etapa (Lujan et al., 2004), entonces conocer qué experiencias académicas tiñeron las vivencias de los ingresantes durante el nivel medio y qué expectativas proyectan respecto de los nuevos contextos de aprendizaje en la universidad, parecen ser aspectos importantes para ayudarlos a integrarse a esta nueva cultura, para orientarlos en relación con lo que es posible esperar, lo que podemos brindarles en relación con sus expectativas y lo que podemos enseñarles -en el sentido más amplio del término- a valorar respecto de lo deseable y lo posible.

En el marco de lo descripto, específicamente con este estudio nos propusimos: 1) conocer las percepciones de los aspirantes a carreras de ingeniería acerca de los rasgos del contexto que contribuyeron a definir sus aprendizajes durante el nivel medio; 2) describir las expectativas de los aspirantes acerca de los rasgos del contexto académico que les gustaría encontrar en la universidad; 3) comparar las percepciones de los aspirantes acerca de los contextos de aprendizaje del nivel medio con las expectativas sobre los contextos de aprendizaje que les gustaría encontrar en la universidad; 4) sugerir características que debieran reunir las clases a las que asistan estos aspirantes a ingeniería de modo de ampliar las posibilidades de satisfacer sus expectativas, promoviendo así una mayor implicación con sus aprendizajes.

\section{METODOLÓGÍA}

Contexto de surgimiento y diseño de la investigación. El trabajo que presentamos forma parte de una investigación de mayor envergadura llevada a cabo por el Laboratorio de Monitoreo de Inserción de Graduados de la Facultad de Ingeniería de la Universidad Nacional de Río Cuarto, en el marco de un proyecto aprobado por la Agencia Nacional de Promoción Científica y Tecnológica, orientado a identificar factores de riesgo de abandono y potencialidades para el éxito en los ingresantes de esta Facultad. Para 
este trabajo, nos focalizamos en uno de los objetivos integrados al proyecto referido; esto es: conocer las percepciones de los ingresantes sobre los contextos de aprendizaje que vivenciaron durante los estudios en instituciones educativas de nivel medio y sus expectativas respecto de la universidad. Así, seleccionamos algunos de los principales resultados obtenidos, particularmente aquellos que en relación a los objetivos propuestos, resultan de mayor interés en el marco de nuestro trabajo. La investigación desarrollada se enmarca dentro de un enfoque cuantitativo y responde a un diseño no experimental transeccional descriptivo (Hernández-Sampieri et al., 2010).

Participantes. Participaron 137 aspirantes a cursar alguna de las carreras que dicta la Facultad de Ingeniería de la Universidad Nacional de Río Cuarto para el ciclo lectivo 2014. Específicamente participaron ingresantes de Ingeniería Química ( $\mathrm{N}=46)$, Ingeniería en Telecomunicaciones $(\mathrm{N}=25)$, Ingeniería Electricista (N=13) e Ingeniería Mecánica ( $\mathrm{N}=53)$.

Descripción del instrumento. Los datos fueron recabados mediante la administración del IPSE -Inventory of Perceived Study Environment- (Wierstra et al., 1999), un cuestionario de administración colectiva que cuenta con 36 ítems. Las respuestas a los ítems se dan en base a una escala tipo Likert, de 6 puntos, en donde los alumnos marcan el grado de acuerdo o desacuerdo con las afirmaciones expresadas en cada uno de ellos. Los valores más bajos son indicadores de desacuerdo (los números 1,2 y 3 ) y los valores más altos $(4,5$ y 6$)$ indican el grado de acuerdo por parte de los estudiantes con las afirmaciones que se presentan en el ítem. El cero, es la opción numérica equivalente al 'no sé qué responder'. La escala de respuesta tipo Likert es la siguiente:

Tabla 1. Ejemplo de escala de puntuación (Martín, 2014).

\begin{tabular}{|c|c|c|c|c|c|c|}
\hline 1 & 2 & 2 & 4 & 5 & 6 & 0 \\
\hline $\begin{array}{c}\text { No } \\
\text { Estoy totalmente } \\
\text { en desacuerdo }\end{array}$ & & & & $\begin{array}{c}\text { Sí } \\
\text { Estoy } \\
\text { totalmente de } \\
\text { acuerdo }\end{array}$ & No sé \\
\hline
\end{tabular}

EL IPSE está compuesto por dos secciones: una primera sección -o sección A-, indaga acerca de las percepciones de los estudiantes respecto de un determinado contexto de aprendizaje. Una segunda sección -sección B-, indaga las expectativas de los estudiantes; esto es, si les gustaría o no que el rasgo contextual por el que se preguntó en la sección A, estuviera o no presente en el contexto considerado. Cabe precisar que para este estudio, empleamos una versión adaptada del IPSE originalmente validado por Martín (2014). No se modificó la redacción de los ítems sino las referencias a las secciones, A y B. Así, mientras que en la versión original, ambas secciones refieren a un mismo contexto de aprendizaje, en nuestra adaptación, la sección A refiere al contexto de aprendizaje percibido respecto del centro de educación secundaria y la sección $\mathrm{B}$, refiere al contexto esperado en relación con la universidad. Veamos un ejemplo de ítem y de modalidad de puntuación para aclarar las ideas expuestas.

Tabla 2. Ejemplo de ítem y de modalidad de puntuación

\begin{tabular}{|l|l|}
\hline \multicolumn{2}{|l|}{ El profesor espera que los alumnos sepan la lección de memoria. } \\
\hline (a) Esto fue así en el secundario & 4 \\
\hline (b) Me gustaría que fuera así en la universidad & 1 \\
\hline
\end{tabular}

Nótese que, en el ejemplo, el número 4 indicaría que quien responde está de acuerdo en que, en general, los profesores del secundario esperaban que las lecciones se dieran de memoria, aunque en algunas oportunidades no haya sido así. A su vez, el número 1 sugiere que a esta persona no le gustaría que fuera así en la universidad, es decir que está totalmente en desacuerdo con que sus profesores de la universidad esperen que los alumnos sepan las lecciones de memoria.

Los 36 ítems del IPSE conforman 8 escalas relativas a aspectos del contexto; a saber: Interacción/ Implicación, Personalización, Inclusión/Participación, Autorregulación/Individualización, Conexión, Reproducción, Aplicación y Tareas de orientación. A continuación se describen las escalas mencionadas.

Interacción/Implicación. Esta escala está conformada por 4 ítems que atienden a las percepciones de los estudiantes acerca de las formas interactivas de enseñanza que se desarrollan en el contexto de aprendizaje (ejemplo: "Durante las clases, los contenidos de la materia se discuten con los estudiantes"). 
Personalización. Los 5 ítems de esta escala buscan conocer las percepciones de los estudiantes acerca de la distancia socio-emocional percibida respecto del docente en el contexto de la clase (ejemplo: "El profesor muestra interés por los problemas de los estudiantes").

Inclusión/Participación. Los 4 ítems de esta escala refieren, por un lado a las percepciones de los estudiantes sobre la posibilidad que tienen para hacer conocer sus opiniones sobre los contenidos y métodos enseñanza; por otro lado, atienden también a las percepciones acerca de la consideración que esas opiniones reciben durante el desarrollo del curso (ejemplo: "Las ideas y sugerencias de los estudiantes son utilizadas en el curso").

Autorregulación/Individualización. Esta escala está compuesta por 5 ítems que refieren a las percepciones de los estudiantes sobre las posibilidades que tienen para auto-dirigir sus aprendizajes y tomar decisiones en el curso (ejemplo: "Me dan la oportunidad de tomar mis propias decisiones sobre qué aprender").

Conexión. Esta escala está conformada por 4 ítems que atienden a las percepciones de los estudiantes respecto del establecimiento de las relaciones que tienen oportunidad de establecer con los contenidos de una asignatura y el modo en que se espera que lleguen a la construcción de dichas relaciones (ejemplo: "EI profesor espera que los estudiantes conecten por sí mismos los diferentes aspectos de los contenidos de la materia").

Reproducción. Los 6 ítems que componen esta escala refieren a percepciones de los estudiantes acerca del modo de vinculación con el saber que el profesor espera que ellos establezcan durante el cursado de la asignatura. Se trata de percepciones de una vinculación algo arbitraria con los conocimientos a aprender, cuando los alumnos consideran que no promueve la construcción de sentido, estamos hablando de un modo de vinculación que promueve la 'reproducción' de los contenidos (ejemplo: "El profesor espera que los estudiantes aprendan las definiciones de memoria lo más literalmente posible”).

Aplicación. Esta escala está compuesta por 4 ítems que aluden a las percepciones de los estudiantes respecto de las posibilidades del uso del conocimiento o de los contenidos de un curso en diversos contextos (ejemplo: "El profesor espera que los estudiantes traten de aplicar lo que han aprendido").

Tareas de orientación. Los 4 ítems que conforman esta escala indagan acerca de las percepciones de los estudiantes sobre la posibilidad de recibir orientación por parte del profesor con respecto a la estructura, procedimientos y objetivos de enseñanza que se promueven durante el desarrollo de las diferentes tareas de aprendizaje que se les solicitan como parte de su proceso de formación (ejemplo: "Los estudiantes reciben información clara sobre las metas y objetivos del curso").

Antecedentes en el uso del IPSE Inventory of Perceived Study Environment. En investigaciones precedentes, los autores del instrumento (Wierstra et al., 2003 y Wierstra et al., 1999) utilizaron el IPSE para conocer cómo influían las percepciones de los estudiantes en la conformación de los contextos de aprendizaje. Específicamente, se centraron en las preferencias de aprendizaje que tenían estudiantes extranjeros que realizaban actividades de intercambio en diferentes países de Europa. En estos estudios se halló que las percepciones de los estudiantes sobre el ambiente de aprendizaje jugaban un rol central en la interpretación acerca del modo en que se conformaba cada ambiente particular y en la forma que los estudiantes orientaban su actuaciones en el contexto (Könings et al., 2005a).

Könings et al. (2008) y Könings et al. (2007), en sucesivos trabajos, estudiaron las percepciones de alumnos y profesores sobre el contexto de aprendizaje, considerando que el principal objetivo de la educación es generar ambientes poderosos de aprendizaje. Avanzando sobre esta idea, entienden que la construcción de comunidades de aprendizaje, característica de esos ambientes propicios para los aprendizajes, no se genera sólo a partir del diseño, ni de la incorporación de actividades, artefactos o vínculos particulares sino que dependen también y principalmente, del modo en que profesores y estudiantes perciben tales contextos. Específicamente, Könings et al. (2008) elaboraron una versión extendida del cuestionario IPSEE- en Dutch (idioma neerlandés), el cual mide las percepciones de los estudiantes respecto a un ambiente de aprendizaje en particular y sus deseos sobre el diseño (Könings et al., 2011).

Siguiendo esta línea, Picarelli et al. (2006) estudiaron en un grupo de estudiantes de educación secundaria holandeses y flamencos, las posibles relaciones entre los estilos de aprendizaje y el contexto de aprendizaje percibido, mediante la utilización del cuestionario IPSE. En tanto, otros investigadores (Jos Fastré et al., 2010) han considerado dos escalas del IPSE (orientación en las tareas y conexión con el material del curso) para conocer más sobre la percepción de un grupo de alumnos de nivel educativo secundario (asistentes a un programa de Enfermería y Cuidado) y los efectos en evaluaciones basadas en competencias y habilidades de desempeño. 
En Argentina, Martín (2014) estudió las cualidades técnicas de este instrumento. Para ello tradujo el IPSE al español de Argentina y realizó una prueba piloto de este instrumento con el objetivo de valorar su alcance y posibilidad de administración en contextos formales. Entre el 2011 y el 2013, valoró las cualidades técnicas del IPSE para 303 estudiantes argentinos que cursaban estudios superiores. Los hallazgos muestran Alfas de Cronbach aceptables, tanto en la parte $A(.84)$ como en la parte $B(.81)$ del instrumento. La prueba de adecuación muestral KMO (Kaiser-Meyer-Olkin) y prueba de Bartlet con resultados de .85 (parte A) y .80 (parte B), mostraron una correlación alta, lo que sugiere un análisis factorial aceptable y la posibilidad de emplearlo con confianza en el marco de los contextos en los que fue estudiado.

\section{RESULTADOS}

La Tabla 3 expuesta a continuación, sistematiza los datos obtenidos para 137 ingresantes en la Facultad de Ingeniería en cada una de las 8 escalas consideradas por el IPSE (Wierstra et al., 1999 en la versión traducida y adaptada al español por Martín, 2014).

Tabla 3. Valores promedio y desvío estándar para cada una de las escalas que integran el IPSE. Datos para 137 ingresantes en carreras de Ingeniería. UNRC. Año 2014

\begin{tabular}{|l|l|l|l|}
\hline Escalas & Sección & M & D.S. \\
\hline Reproducción & $\mathrm{A}$ & 3,30 & 0,95 \\
& $\mathrm{~B}$ & 3,88 & 0,84 \\
\hline Conexión & $\mathrm{A}$ & 3,32 & 1,23 \\
& $\mathrm{~B}$ & 4,32 & 1,23 \\
\hline Aplicación & $\mathrm{A}$ & 4,30 & 0,83 \\
& $\mathrm{~B}$ & 5,33 & 0,72 \\
\hline Interacción/Implicación & $\mathrm{A}$ & 4,44 & 0,92 \\
& $\mathrm{~B}$ & 5,23 & 0,87 \\
\hline Personalización & $\mathrm{A}$ & 4,58 & 0,90 \\
& $\mathrm{~B}$ & 5,20 & 0,70 \\
\hline Inclusión/Participación & $\mathrm{A}$ & 3,74 & 0,79 \\
& $\mathrm{~B}$ & 3,95 & 0,93 \\
\hline Autorregulación/Individualización & $\mathrm{A}$ & 2,97 & 0,89 \\
& $\mathrm{~B}$ & 3,63 & 1,02 \\
\hline Tareas de Orientación & $\mathrm{A}$ & 4,10 & 0,98 \\
& $\mathrm{~B}$ & 5,03 & 0,94 \\
\hline
\end{tabular}

Considerando las puntuaciones que este grupo de ingresantes obtuvo en la sección B del instrumento expectativas por los contextos de aprendizaje que les gustaría experimentar en la universidad-, los resultados muestran que los valores más altos de la escala (comprendidos entre 5 y 6) se concentran particularmente en cuatro escalas: aplicación, interacción/implicación, personalización y tareas de orientación. Esto supone que, en general este grupo de ingresantes espera participar de contextos de aprendizaje que promuevan la transferencia y el uso del conocimiento, favorezcan las interacciones entre alumnos entre sí y con los docentes, ayuden a reducir las distancias percibidas entre el profesor y los estudiantes y brinden orientaciones claras por parte del profesor respecto de la estructura, los procedimientos y los objetivos de enseñanza que se promueven durante el desarrollo de las diferentes tareas de aprendizaje que se les propone como parte de su proceso de formación. Los resultados discriminados por carrera no mostraron variaciones atendibles en los aspectos aquí referidos.

En cuanto a las percepciones de los estudiantes sobre los rasgos del contexto de aprendizaje relativos a sus colegios de educación secundaria (sección A), los resultados no muestran puntuaciones altas (esto es, valores comprendidos entre 5 y 6 ) en ninguna de las variables consideradas por el instrumento. En general, se trata de valores medios (entre 3 y 4 ) o bien de valores medio-altos (entre 4 y 5). En esta sección es en la única que se obtiene un valor considerado medio-bajo (comprendido entre 2 y 3 ) relativo a la escala autorregulación/individualización lo que sugiere que este grupo de ingresantes en particular coincide en comunicar insatisfacción respecto de las posibilidades que tuvieron para autorregular sus aprendizajes y tomar decisiones en el curso de sus estudios en el colegio de educación secundaria.Finalmente, comprobada la bondad de ajuste de los datos a lo teóricamente esperado (distribución normal según la prueba Kolmogorov-Smirnov), efectuamos el análisis de la diferencia entre las medias obtenidas por los ingresantes en la sección A y en la sección B del instrumento -según la prueba t de Student para muestras relacionadas-, advirtiéndose diferencias significativas en todas las escalas tal y como se observa en la Tabla 4 expuesta a continuación. 
Tabla 4. Prueba de muestras relacionadas según prueba t. Datos para 137 alumnos aspirantes a carreras de ingeniería. UNRC. Año 2014.

\begin{tabular}{|c|c|c|c|c|c|c|c|c|c|}
\hline & \multicolumn{5}{|c|}{ Diferencias relacionadas } & \multirow{3}{*}{$t$} & \multirow{3}{*}{$\mathrm{gl}$} & \multirow{3}{*}{$\begin{array}{l}\text { Sig. } \\
\text { (bilat.) }\end{array}$} \\
\hline & & \multirow[t]{2}{*}{ Media } & \multirow[t]{2}{*}{ Desv. típ. } & \multirow{2}{*}{$\begin{array}{l}\text { Error típ. } \\
\text { de la } \\
\text { media }\end{array}$} & \multicolumn{2}{|c|}{$\begin{array}{l}95 \% \text { intervalos de } \\
\text { confianza para la } \\
\text { diferencia }\end{array}$} & & & \\
\hline & & & & & Inferior & Superior & & & \\
\hline Par 1 & $\begin{array}{l}\text { A-reproducción } \\
\text { B-reproducción }\end{array}$ &,- 58151 & ,91625 & ,07828 &,- 73631 &,- 42670 & $-7,429$ & 136 & ,000 \\
\hline Par 2 & $\begin{array}{l}\text { A-conexión } \\
\text { B-conexión }\end{array}$ &,- 99453 & 1,22192 & ,10440 & $-1,20097$ &,- 78808 & $-9,527$ & 136 & ,000 \\
\hline Par 3 & $\begin{array}{l}\text { A-aplicación } \\
\text { B-aplicación }\end{array}$ & $1,02737^{-}$ & ,89251 & ,07625 & $-1,17817$ &,- 87658 & $-13,473$ & 136 & ,000 \\
\hline Par 4 & $\begin{array}{l}\text { A-implicación } \\
\text { B-implicación }\end{array}$ &,- 78832 & 1,02894 & ,08791 &,- 96217 &,- 61448 & $-8,968$ & 136 & ,000 \\
\hline Par 5 & $\begin{array}{l}\text { A-personalización } \\
\text { B-personalización }\end{array}$ &,- 61314 & ,88391 & ,07552 &,- 76248 &,- 46380 & $-8,119$ & 136 & ,000 \\
\hline Par 6 & $\begin{array}{l}\text { A-participación } \\
\text { B-participación }\end{array}$ & ,33759 & ,76372 & ,06525 & ,20856 & ,46662 & 5,174 & 136 &, 000 \\
\hline Par 7 & $\begin{array}{l}\text { A-individualización } \\
\text { B-individualización }\end{array}$ &,- 66131 & ,94762 & ,08096 &,- 82142 &,- 50121 & $-8,168$ & 136 & ,000 \\
\hline Par 8 & $\begin{array}{l}\text { A-tareas de orient. } \\
\text { B-tareas de orient. }\end{array}$ &,- 93431 & 1,06941 & ,09137 & $-1,11499$ &,- 75363 & $-10,226$ & 136 &, 000 \\
\hline
\end{tabular}

Si se analizan los valores de las Tabla 3 y 4, puede advertirse que las puntuaciones obtenidas por los aspirantes a ingeniería en relación con las expectativas acerca de los diversos rasgos del contexto de aprendizaje que les gustaría experimentar en la universidad (sección B), son más altas en comparación con las percepciones de esos mismos rasgos en el nivel medio que cursaron (sección A). Los resultados se orientan en el sentido que supone la teoría, en todas las escalas excepto en la escala de reproducción. Para esta escala en particular, es llamativo que las expectativas de un aprendizaje por reproducción 'superen' por así decirlo- a las percepciones de este mismo rasgo en relación al centro de nivel medio. Esta aparente contradicción con lo teóricamente esperado e incluso con los valores elevados obtenidos en el resto de las escalas -como autorregulación/individualización o personalización-, sugiere la necesidad de profundizar en este aspecto en particular, indagando quizás el sentido de las representaciones construidas por estos jóvenes respecto del valor personal y social de rasgos contextuales como la autonomía, la participación y la creatividad frente al reproduccionismo y la pasividad.

\section{DISCUSIÓN}

Retomando los supuestos y objetivos que orientaron el estudio realizado, es posible efectuar algunas consideraciones acerca de los resultados obtenidos. Los hallazgos de este estudio permiten considerar que las percepciones de los aspirantes a carreras de ingeniería acerca de los rasgos que definen a los contextos de aprendizaje del nivel medio y las expectativas acerca de los contextos académicos que les gustaría encontrar en el nivel superior, no se expresan con igual intensidad. Así, los únicos valores promedio altos (comprendidos entre 5 y 6 ) fueron obtenidos en relación con las expectativas de los aspirantes sobre rasgos de contextos de aprendizaje que les gustaría hallar en la universidad. Específicamente, estas expectativas elevadas se manifiestan en relación con los rasgos de aplicación, interacción/implicación, personalización y orientación de la tarea consideradas por el instrumento; todos ellos, rasgos característicos de contextos promisorios para la conformación de comunidades de aprendizaje potencialmente efectivas para el logro de las metas educativas (Martín, 2014). Así, estos resultados sugieren que los ingresantes valorarían especialmente formar parte de contextos académicos en los que se promueva la construcción de aprendizajes con sentido, donde se propongan tareas dinámicas que fomenten su participación, procesos de interacción e implicación; contextos en los que procesos de feedback promueven mayores cuotas de autonomía y donde las orientaciones brindadas por el profesor ayuden a autorregular los recursos internos y externos con que cuentan para aprender.

¿Cómo podemos aprovechar estos hallazgos para el diseño de contextos promisorios para el aprendizaje? De acuerdo con Könings et al. (2008), si antes de 'entrar' en un nuevo entorno de aprendizaje los estudiantes comunican expectativas altas por encontrar allí rasgos como interacción/implicación, 
inclusión/participación, personalización, etc., entonces es más probable que estas expectativas se relacionen luego con percepciones positivas de esos mismos rasgos contextuales al pasar el tiempo. Vinculando estos planteamientos con los resultados de nuestro estudio, tendríamos entonces un grupo con altas expectativas respecto a los rasgos contextuales promisorios para la motivación y el aprendizaje. Así, de acuerdo con los hallazgos obtenidos, este grupo de ingresantes en particular esperaría participar de experiencias de aprendizaje que atiendan a la transferencia y al uso de conocimientos en diversos contextos (aplicación), donde primen formas interactivas de enseñanza capaces de promover intercambios, diálogo e implicación por parte de alumnos y docentes (interacción/implicación), clases en las que se perciba a profesores interesados por sus estudiantes, atentos a sus fortalezas y preocupados por sus dificultades (personalización), contextos en donde las demandas de las tareas requeridas -como parte del proceso de formación- sean claras, con objetivos explícitos y orientaciones pedagógicas que sugieran un rumbo interesante y permitan construir aprendizajes con sentido (orientación de la tarea).

Los resultados expuestos plantean entonces un desafío. Dependerá de nosotros y de nuestra participación como docentes o como investigadores educacionales, afrontarlo en el diseño propuestas pedagógicas que efectivamente incluyan todas o algunas de estas características deseables para cualquier contexto de aprendizaje.

\section{CONCLUSIONES}

Los resultados expuestos, su análisis y discusión, nos permiten arribar a algunas conclusiones y sugerencias que intentan echar luces en el diseño de contextos académicos potencialmente promotores de compromiso, implicación y aprendizajes con sentido en la universidad.

Importancia de atender a las expectativas de los estudiantes. De acuerdo con los marcos teóricos en los que nos posicionamos -Könings et al. (2005a); Könings et al. (2005b); Martín, (2014); Rinaudo, (2014); entre otros-, estudiantes y docentes significan e interpretan de un modo particular lo que ocurre en los salones de clase. En general, para los autores consultados, la congruencia entre las interpretaciones de alumnos y docentes es fundamental en tanto favorecería la concreción de aprendizajes más efectivos por parte de los estudiantes al promover prácticas pedagógicas sensibles a sus intereses, preferencias, características, expectativas y experiencias. En el caso de nuestro estudio, los valores más altos logrados en las escalas utilizadas, sugieren que la posibilidad de transferir y aplicar los conocimientos académicos a contextos diversos, de hacer un uso inteligente del saber cómo herramienta simbólica y la oportunidad de conformar una red de interacciones fluidas entre pares y con el profesorado, son algunos de los principales rasgos del contexto que estos aspirantes a ingeniería quisieran encontrar en la universidad. Estos rasgos situacionales, coinciden a su vez con lo que algunos teóricos de la motivación y del aprendizaje destacan como atributos de un contexto promisorio para promover compromiso e implicación por parte de los estudiantes y las posibilidades de obtener, por lo mismo, resultados óptimos (Alexander, 2006; Huertas et al., 2001; Ryan y Deci, 2000, entre otros).

El feedback sobre percepciones del contexto como un modo de promover mayor congruencia entre las interpretaciones situacionales de estudiantes y docentes. Entendemos que el hecho de responder a un cuestionario sobre percepciones y expectativas del contexto académico -como el utilizado en este trabajo-, promovió en los ingresantes en carreras de ingeniería una instancia de reflexión acerca de sus vivencias como estudiantes del nivel medio y sus proyecciones y expectativas futuras como alumnos universitarios. En próximas investigaciones sería interesante en tal sentido, dar un paso más, de modo que docentes y estudiantes participen activamente en instancias de feedback en torno a los resultados obtenidos, con el propósito de avanzar hacia la construcción de una mayor congruencia entre las interpretaciones situaciones de unos y de otros; en otras palabras, entre lo que esperan encontrar los alumnos y lo que piensan proponer los docentes.

Conveniencia de complementar los resultados obtenidos con análisis cualitativos que permitan profundizar en el sentido de los datos y amplíen así el alcance del trabajo en su conjunto. Si bien los resultados obtenidos en este trabajo se orientan a considerar la importancia de atender a las interrelaciones establecidas entre aspectos personales -percepciones, expectativas- y contextuales en los aprendizajes académicos, parece oportuno completar su enfoque con datos y análisis provenientes de tradiciones cualitativas. Tal y como lo destaca Paoloni (2010) en una minuciosa revisión de literatura especializada, una tendencia metodológica en investigación educacional alude al hecho de proponer estudios multi-métodos que combinen una variedad de métodos de investigación, fuentes de información y herramientas de análisis. Así, pensamos que esta es una sugerencia especialmente prometedora para quienes se interesen por el estudio de las percepciones y expectativas del contexto académico. 
En definitiva, entendemos que, con sus fortalezas y limitaciones, el estudio que presentamos y los resultados obtenidos brindan aportes y sugerencias interesantes para quienes desean incursionar o bien profundizar en el estudio de las percepciones y expectativas sobre contextos de aprendizaje; ello por al menos tres razones: propone marcos teóricos actuales que atienden a la dinámica establecida entre persona y situación en contextos de aprendizaje; propone un modo de aproximarse a dicha dinámica; sugiere senderos por donde podría discurrir futura investigación al respecto.

\section{REFERENCIAS}

Alexander, P., Psychology in Learning and Instruction. Ohio: Pearson Merril Prentice Hall. (2006)

Bransford, J., Brown, A. y R. Cocking, How people learn. Brain, Mind, Experience and School. National Academy Press, Washington, Estados Unidos (2003)

Cole, M., Psicología Cultural: una disciplina del pasado y del futuro. Morata, Madrid, España (1999)

De Corte, E., Marying theory building and the improvementt of school practice: a permanent challenge for instructional psychology, Learning and Instruction, 10: 249-266 (2000)

García, J. C., M. Gonzáles y A. Zanfrillo, Desgranamiento universitario: perspectiva estudiantil en ingeniería, Actas del XI Coloquio Internacional de Gestión Universitaria de América del Sur. Universidad de Santa Catarina, Florianopolis, Brasil (2011)

Hernández Sampieri R., Fernández C. y P. Baptista, Metodología de la investigación, McGraw- Hill, México (2010)

Huertas, J. A., Montero, I. y S. Pironio, La interacción en el aula. Aprender con los demás. Buenos Aires: Aique (2001)

Jos Fastré, G.M., van der Klink, M. y J.J.G. van Merriënboer, The effects of performance-based assessment criteria on student performance and self-assessment skills, Advances Health Sciences Education Theory Pract, 15(4), 517-532 (2010)

Könings, K. D., Brand-Gruwel, S. y J. van Merriënboer, Towards more powerful learning environments through combining the perspectives of designers, teachers, and students, British Journal of Educational Psychology, 75: 645-660 (2005a)

Könings, K. D., Brand-Gruwel, S. y J. van Merriënboer, Combining different perspectives on a learning environment: students' and teachers' perceptions. Poster presented at the Conference on Advancing Teacher Learning, Ascona, Switzerland, June. (2005b)

Könings, K. D., S. Brand-Gruwel, J. J. van Merriënboer y N. Broers, Does a new learning environment come up to students' expectations? A longitudinal study, Journal of Educational Psychology, 100: 535-548 (2008)

Könings, K. D., T. Seidel, S. Brand-Gruwel y J. van Merriënboer, Students' and Teachers' Perceptions of Education: Differences in Perspectives, Actas del $14^{\circ}$ Biennial Conference for Research on Learning and Instruction, Exeter, Inglaterra (2011)

Könings, K. D., M. Van Zundert, S. Brand-Gruwell y J. Van Merriënboer, Participatory design in secondary education: Is it a good idea? Students' and teachers' opinions on its desirability and feasibility, Educational Studies, 33(4): 445-465 (2007)

Luján, S., A. Montenegro, L. Ponti, A. Sánchez Malo, C. Savino, R. Vasquetto y G. Vélez, Aprendiendo a ser estudiante universitario, Módulo Problemática Universitaria y Sociedad, Secretaría Académica de la Universidad Nacional de Río Cuarto, Río Cuarto, Argentina (2004). (pp.: 4-22) Disponible en: http://www.unrc.edu.ar/unrc/academica/pdf/folleto-aprendiendo-ser-est.pdf

Martín, R., Comunidades de aprendizaje en contextos formales y no formales. Tesis doctoral inédita, Universidad Nacional de San Luis, Argentina (2014)

Panaia, M., Perspectiva futura para los ingenieros. Perfiles, Boletín del Laboratorio de Monitoreo de Inserción de Graduados de la UTN Facultad Regional Avellaneda, 2(3): 1-2 (2007). Disponible en http://www.fra.utn.edu.ar/mig/documentos/perfiles_3_web.pdf (22/03/2015) 
Panaia, M., Trayectorias de Graduados y estudiantes de Ingeniería. Editorial Biblios, Buenos Aires, Argentina (2011a)

Panaia, M., Dejar la Universidad ¿Decisión o imprevisto? Itinerarios, Boletín del Laboratorio de Monitoreo de Inserción de Graduados de la Facultad de Ingeniería, 12: 2-4, Universidad Nacional de Río Cuarto, Argentina (2011b). Disponible en http://www.ing.unrc.edu.ar/laboratorios/mig_rio4/archivos/12-boletin-junio2011.pdf (22/03/2015)

Paoloni, Paola V., Las tareas académicas como contextos promisorios para la motivación y el aprendizaje. En P. V. Paoloni, M. C. Rinaudo, D. Donolo, A. González Fernández y N. Rosselli. Estudios sobre motivación: enfoques, resultados, lineamientos para acciones futuras. Editorial de la Universidad Nacional de Río Cuarto (2010)

Paoloni, P. V. y J. Moreno, Facebook en el andamiaje socioemocional de ingresantes universitarios. Potencialidades para la conformación de comunidades online. Actas del 6to Seminario Internacional de Educación a Distancia. Universidad Nacional de Cuyo (2013). Disponible en http://www.uncu.edu.ar/seminario_rueda/upload/t66.pdf (22/03/2015)

Paoloni, P. V., A. Chiecher y R. Martín, Percepciones y expectativas por contextos de aprendizaje. Un estudio con ingresantes en carreras de Ingenieria, Actas del Congreso Argentino de Ingeniería -CADI-, Tucumán, Argentina. Publicado en CD: ISBN 978-987-1662-51-7 (septiembre 2014)

Picarelli, A., Slaats, M., Bouhuijs, P. A. J. y J. D. Vermunt, Leerstijl en leeromgeving in het voortgezet onderwijs: Nederland en vlaanderen vergeleken [Learning style and learning environment in secondary education: The Netherlands and flanders compared]. Pedagogische Studiën, 83, 139-155 (2006)

Rinaudo, M., Estudios sobre los contextos de aprendizaje. Arenas y fronteras. En P.V. Paoloni, M. C. Rinaudo y A. González Fernández (comps.) Cuestiones en Psicología Educacional: cuestiones teóricas, metodológicas y estudios de campo. Sociedad Latinoamericana de Comunicación Social (SLCS), (pp: 163205) Tenerife, España (2014). Disponible en http://www.cuadernosartesanos.org/2014/cde01.pdf $(22 / 03 / 2015)$

Ryan, R. y E. Deci, Self-Determination Theory and the Facilitation of Intrinsic Motivation, Social Development, and Well-Being. American Psychologist, 55 (1), 68-78 (2000)

Vélez, G., Ingresar a la Universidad. Aprender el oficio de estudiante universitario. Colección de Cuadernillos de Actualización para pensar la enseñanza Universitaria, año 2(1): 5-14 (2005). Disponible en http://www.unrc.edu.ar/unrc/academica/pdf/cuadernillo02.pdf (22/03/2015)

Wierstra, R., G. Kanselaar, J.L. Linden, L. van der, H. Lodewijs y J. Vermunt, The impact of the university context on European students'learning strategies and learning environment preferences, Higher Education, 45: 503-523 (2003)

Wierstra, R., G. Kanselaar, J. van der Linden y H. Lodeewijks, Learning Environment Perceptions of European University Student, Learning Environments Research,1(2): 79-98 (1999) 